\title{
EFEITO DA ADIÇÃO DE NANOFIBRILAS DE CELULOSE DE Eucalyptus spp. NA PRODUÇÃO DE PAPÉIS DE FIBRAS RECICLADAS
}

\author{
EFFECT OF THE ADDITION OF NANOFIBRILS OF EUcalyptus spp. CELLULOSE IN THE \\ PRODUCTION OF PAPERS OF RECYCLED FIBERS
}

\author{
Gustavo Spiering Zanol ${ }^{1}$, Henrique Römer Schulz'2, Andrey Pereira Acosta ${ }^{3}$, \\ Gabriel Valim Cardoso ${ }^{4}$
}

\author{
${ }_{1}^{1}$ Instituto Federal Sul-Rio-Grandense, Pelotas, Rio Grande do Sul, Brasil-gustavo-zanol@hotmail.com \\ 2, 3, 4 Universidade Federal de Pelotas, Pelotas, Rio Grande do Sul, Brasil - henriqueshulz09@hotmail.com, \\ andreysvp@gmail.com \& gabriel.valim.cardoso@gmail.com
}

\section{RESUMO}

Tendo em vista um aumento no apelo ambiental, a evolução na produção de papéis reciclados de aparas é motivada pela necessidade de reduzir custos e minimizar o impacto causado por estes produtos, bem como, a necessidade de modificações que visem mudanças nas propriedades físico-mecânicas, como por exemplo, a inserção de materiais nano na produção do papel. Sendo assim, o presente estudo teve como objetivo, avaliar as propriedades tecnológicas de papéis reciclados, produzidos com a adição de diferentes concentrações de nanofibrilas de celulose (NFC), de Eucalyptus spp. Produziu-se papéis com $60 \mathrm{~g} / \mathrm{cm}^{2}$ de gramatura, adicionando-se diferentes concentrações de nanofibrilas de celulose nos papéis, a fim de avaliar o efeito destas na união das fibras e nas propriedades tecnológicas dos papéis reciclados, para isso foi feita a caracterização por MEV dos papéis sem adição e com $16 \%$ de concentração de NFC. Foram efetuados testes de índice de tração, elongamento, índice de estouro, espessura, passagem do ar (Gurley) e gramatura. Com a análise dos resultados, foi possível concluir que a maior parte das propriedades tecnológicas apresentou melhora com o aumento na concentração de nanofibrilas. Para resistência a tração em que houve um acréscimo de $266 \%$, para elongamento com um ganho de $308 \%$ e resistência ao estouro um incremento de $407 \%$. Assim a inserção de nanocelulose demonstra ser um bom aditivo para a produção de papéis, aumentando as propriedades físicas e mecânicas dos papéis.

PALAVRAS-CHAVE: Fibra curta, MEV, Nanocelulose, Tração.

\section{ABSTRACT}

In view of an increase in environmental appeal, the evolution in the production of recycled scrap paper is motivated by the need to reduce costs and minimize the impact caused by these products, as well as the need for modifications aimed at changes in physical and mechanical properties, such as the insertion of nano materials in paper production. Thus, the present study aimed to evaluate the technological properties of recycled papers, produced with the addition of different concentrations of Eucalyptus spp. Paper with $60 \mathrm{~g} / \mathrm{cm}^{2}$ of weight was produced, adding different concentrations of cellulose nanofibrils in the papers, in order to evaluate their effect on fiber bonding and on the technological properties of recycled papers. of papers without addition and with $16 \%$ NFC concentration. Tensile index, elongation, burst index, thickness, air passage (Gurley) and weight tests were performed. With the analysis of the results, it was possible to conclude that most of the technological properties showed improvement, with the increase of nanofibrils concentration, for tensile strength in which there was an increase of $266 \%$, for elongation with a gain of $308 \%$ and strength. overflow an increase of $407 \%$. Thus, the insertion of nanocellulose proves to be a good additive for paper production, increasing the physical and mechanical properties of paper.

KEYWORDS: Short fiber, MEV, Nanocellulose, Traction. 


\section{INTRODUÇÃO}

Para a fabricação de papéis, o gênero com grande destaque é o Eucalyptus. Sendo um gênero que apresenta resultados satisfatórios na produção de papel tanto de fibra virgem como de fibra reciclada (SCHULZ, 2019).

Com a utilização deste gênero, o setor de celulose e papel tem crescido anualmente constantemente e ocupado posições expressivas no mercado nacional e internacional (BRACELPA, 2014). As indústrias de celulose e papel apresentam um perfil sustentável, pois a matériaprima é considerada como recurso renovável, sendo oriundo desse os produtos recicláveis.

Sob uma perspectiva de conscientização ambiental, existe uma tendência de aumento de produção de papéis reciclados, assim, a evolução no consumo de aparas é motivada pela necessidade de reduzir custos de produção e minimizar o impacto de resíduos sólidos. 0 reaproveitamento de materiais já utilizados viabiliza o uso de matéria-prima de menor valor agregado e o desenvolvimento de novas tecnologias (MANFREDI, 2012).

A fabricação de produtos reciclados causa contestações de qualidade dentro da sociedade, as quais possuem abrangentes motivos, o papel reciclado, possui algumas limitações técnicas, que comprometem a qualidade dos produtos gerados por fibras que cumpriram um ciclo de utilização e sofreram transformações físicas e químicas em sua estrutura (CASTANHO \& OLIVEIRA, 2000).

Essas transformações tornam atraente o uso de nanofibrilas como promissor para aplicação como agente de reforço (AARSTAD et al., 2017).

As nanofibrilas de celulose são incorporadas ao papel com o objetivo de aumentar as propriedades e dar um melhor acabamento, pois devido ao tamanho das fibras tem o potencial de ocupar pequenos espaços vazios e, proporcionar um melhor entrelaçamento das fibras, tornando-se um provável aditivo, melhorando suas propriedades físicas e a resistência das propriedades mecânicas (POTULSKI et al., 2014). Com tais modificações se faz necessário o conhecimento dos parâmetros tecnológicos dos papéis.

Sendo assim, o presente trabalho teve como objetivo, avaliar as propriedades físicas e mecânicas de papéis reciclados obtidos de aparas de papel branqueado e papel não branqueado kraft com diferentes concentrações de nanofibrila de celulose (NFC) de Eucalyptus spp.

\section{MATERIAL E MÉTODOS}

\section{Matéria prima}

Os materiais utilizados para a pesquisa foram fibras de celulose branqueada de madeira de Eucalyptus spp, para produção das nanofibrilas, e fibras recicladas obtidas de aparas de papel branqueado e de papel não branqueado kraft.

\section{Produção das nanofibrilas e papéis}

Para a produção das nanofibrilas de celulose, foi realizada em refinador de discos tipo Bauer modelo MD3000, utilizando fibras de celulose branqueada de Eucalyptus spp. O processo mecânico de nanofibrilação consistiu em refinar as fibras (tratamento mecânico direcionado a polpa celulósica com objetivo de ampliar a capacidade de entrelaçamento das fibras) por um período de 3 horas com consistência de $2 \%$. Após a produção, as nanofibrilas foram mantidas sob refrigeração na consistência de 2,2\%, para posterior aplicação nos papéis e uma parte foi utilizada para Microscopia Eletrônica Varredura um microscópio eletrônico de varredura (MEV).

Definiu-se a formação de folhas de papel branqueado e não branqueado kraft, com gramatura de $60 \mathrm{~g} / \mathrm{m}^{2}$, nas quais foram adicionadas concentrações de nanofibrilas de 0, 2, 4, 8 e 16, 32 e $64 \%$.

Em seguida, quantificado o correspondente a 24 gramas secas de papel branqueado e papel não branqueado kraft, as quais ficaram imersas em 2 litros de água destilada por 48 horas, garantindo a hidratação total das fibras para posterior individualização.

Para a desagregação das fibras foi realizado o processo de desintegração das fibras utilizando um desintegrador automático de acordo com as normas técnicas ISO 5263-1 e 5263-2, permanecendo por 10.000 rotações para desagregação completa.

As regulagens da consistência mantiveram-se ambas polpas desintegradas em dois galões distintos $e$ adicionando ao total 8 litros de água destilada em cada galão, mantendo assim a consistência de $0,3 \%$, calculado pela Equação 1.

$$
\mathrm{Cs}=\frac{(\mathrm{g})}{(\mathrm{ml})} \times 100
$$

Em que: $\mathrm{Cs}$ = consistência do material em porcentagem; $\mathrm{g}=$ material em gramas secas; $\mathrm{ml}=$ quantidade de água para a diluição do material.

A mistura das nanofibrilas foi realizada no desintegrador, onde foram adicionados as nanofibrilas nas diferentes concentrações, o volume correspondente de polpa de papel (não branqueado kraft ou branqueado) e 
1,5 L de água, agitando-se por 10.000 rotações.

Para a formação das folhas de papel (delineamento expresso na Tabela 1) foi utilizado equipamento formador folhas de laboratório convencional, modelo $\mathrm{FF} / \mathrm{C}$, para fabricação de folhas de acordo com a norma TAPPI T 20.

Tabela 1. Concentrações de nanofibrilas de celulose em papéis de $60 \mathrm{~g} / \mathrm{m}^{2}$.

\begin{tabular}{cccc}
\hline NFC $\%$ & Gramas & Polpa $(\mathbf{m L})$ & Água (I) \\
\hline 0 & - & 400 & 1,5 \\
2 & 0,02 & 392 & 1,5 \\
4 & 0,05 & 384 & 1,5 \\
8 & 0,10 & 368 & 1,5 \\
16 & 0,19 & 336 & 1,5 \\
32 & 0,38 & 272 & 1,5 \\
64 & 0,77 & 144 & 1,5 \\
\hline
\end{tabular}

Após a formação da folha, o material foi levado para prensa pneumática para folhas de laboratório Regmed, modelo SP-21b, de acordo com ABNT NBR ISO 5269/1 e TAPPI T 205

Por fim, levado a câmara climatizada para a climatização das folhas de papel, adquirindo teor de umidade e peso constante, segundo a norma ISO 187:1990.

Os papéis produzidos sem adição e com adição de $16 \%$ de nanofibrilas foram caracterizados por MEV. Para isso, foram preparadas as superfícies secas dos corpos de prova com recobrimento por pó de ouro, o qual recobre a superfície do papel, funcionando como um condutor para a passagem de feixes de elétrons, assim foram obtidas micrografias com aumento de 100 e 30.000 vezes.

\section{Caracterização das propriedades tecnológicas dos papéis}

Para as propriedades físicas e mecânicas dos papéis foram feitas as análises de índice de estouro, resistência ao ar de Gurley, espessura, índice de tração, elongamento e gramatura, seguindo as normas ISO, conforme a Tabela 2.

Tabela 2. Propriedades analisadas com base nas respectivas normas.

\begin{tabular}{cc}
\hline Propriedades & Normas \\
\hline Teor de umidade & ISO 534: 2005 \\
Índice de estouro & ISO 2758: 2014 \\
Índice de tração & ISO 1924-2: 2008 \\
Resistência ao ar de Gurley & ISO 5636-5: 2013 \\
Elongamento & ISO 1924-3: 2008 \\
Gramatura & ISO 536: 1995 \\
Espessura & ISO 534: 2005 \\
\hline
\end{tabular}

\section{Teor de Umidade}

Foi pedado 2 gramas úmidas de amostra de cada papel em uma placa de petri, realizado em duplicata, foi acondicionado em estufa por 24 horas, retirado da estufa e pesado novamente, descontando o peso da placa é possível obter a massa da amostra seca, dessa forma aplicando na Equação 2.

$$
\mathrm{TU}=\frac{(\mathrm{MU}-\mathrm{MS})}{\mathrm{MS}} \times 100
$$

Em que: $\mathrm{TU}=$ teor de umidade (\%); $\mathrm{MU}=$ massa $\mathrm{da}$ amostra úmida (g); MS = massa da amostra seca (g).

\section{Índice de Estouro}

Foram confeccionados os corpos de prova, os quais foram aplicados no equipamento $L \& W$ teste de resistência ao estouro, conforme a norma ISO 2758: 2014.

\section{Índice de tração e elongamento}

Foi utilizado uma guilhotina para seccionar os papéis branqueados e não branqueados kraft, confeccionando corpos de prova com $15 \mathrm{~mm}$ de largura, assim foram testados no dinamômetro, conforme as normas ISO 1924/2: 2008 e ISO 1924/3: 2008.

\section{Resistência ao ar de Gurley}

Foram confeccionados os corpos de prova os quais foram aplicados no L\&W permeância ao ar, conforme ISO 5636-5: 2013.

\section{Gramatura}

Foi encontrado a massa das amostras através de pesagem em balança analítica, calculado o peso médio da Equação 3, posteriormente, essa massa foi multiplicada por um fator de correção, calculando assim a gramatura dos papéis por meio da Equação 4, conforme ISO 536: 1995.

$$
\begin{gathered}
M M=\frac{M T}{N F} \\
G=P M \times 50
\end{gathered}
$$

Em que: $\mathrm{MM}=$ peso médio; $\mathrm{MT}=$ massa total; $\mathrm{NF}=$ número de folhas; $\mathrm{G}=$ gramatura. 


\section{Espessura}

A espessura foi medida com auxílio de um micrômetro aferindo em 2 folhas fazendo 6 medidas em pontos diferentes, sendo um dos pontos no centro da folha, conforme ISO 534: 2005.

\section{Grau Schopper Riegler}

Para a determinação do Grau Schopper Riegler, efetuou-se o processo de preparação da amostra, determinação do teor de umidade dos papéis e seguindo os passos de pesagem, hidratação, desintegração, consistência e mistura, como realizado para a formação dos papéis, após todo o processo foi determinado o Grau Schopper Riegler, seguindo a norma ISO 5267- 1:1999.

\section{Análise estatística}

Utilizou-se o delineamento inteiramente casualizado (DIC) para os papéis com e sem adição de NFC. A homogeneidade dos resultados foi avaliada por meio do teste Boxplot, em que estes se mostraram satisfatórios para aplicação dos testes paramétricos. Em caso de rejeição da hipótese nula, foram realizados os testes de Tukey e $F$ em nível de $5 \%$ de significância de erro. A correlação entre as variáveis de interesse no estudo foi verificada por meio da correlação de Pearson, em $5 \%$ de probabilidade de erro.

\section{RESULTADOS E DISCUSSÃO}

\section{Microscopia das nanofibrilas de celulose}

A figura 1 apresenta a microscopia que corresponde as nanofibrilas, comprovado pelas dimensões observadas em escala nanometricas de 28 a 74 nanometros, caracterizado por microscopia eletrônica de varredura (MEV), ao considerar celulose nanofibrilada como nanoestruturas que apresentam diâmetro menores que $100 \mathrm{~nm}$ (SEHAQUI et al., 2011).

Com a microscopia eletrônica de varredura (MEV) foi possível notar as características das nanofibrilas de celulose que, por sua vez, possuem diâmetros na escala de nanômetros e comprimento alongado, obtido pelo tratamento mecânico de desfibrilação.

O tratamento mecânico de desfibrilação efetua alterações que não podem ser mudadas nas fibras, como a redução da cristalinidade e da viscosidade (HAl et al., 2013). Este processo mecânico de refinação efetua alterações estruturais das fibras, o refino faz as fibras se conformarem melhor entre si, dando um aumento a área superficial, e, portanto, subtraindo a densidade, o volume específico e a porosidade do papel (BIERMANN, 1996).

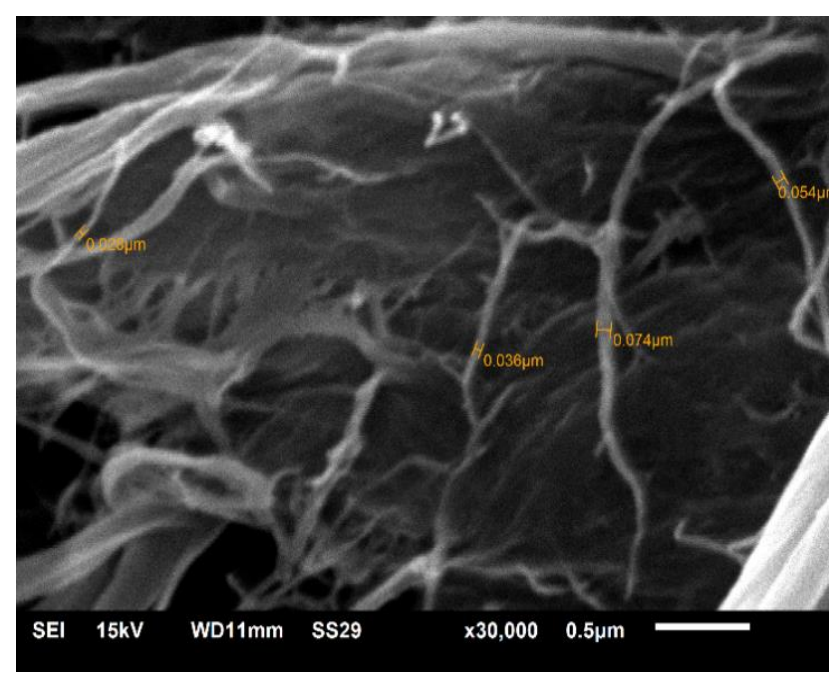

Figura 1. Microscopia eletrônica das fibras aumento 30.000 vezes / escala $0,5 \mu \mathrm{m}$.

\section{Microscopia eletrônica de varredura}

As Figuras 2 e 3 apresentam micrografias obtidas por MEV dos papéis branqueados e não branqueados kraft sem e com adição de $16 \%$ de nanofibrilas, respectivamente.
(A) sem adição de NFC

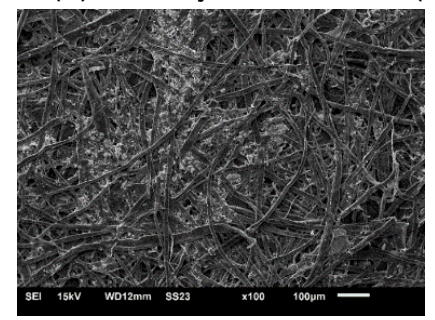

(B) com $16 \%$ de adição de NFC

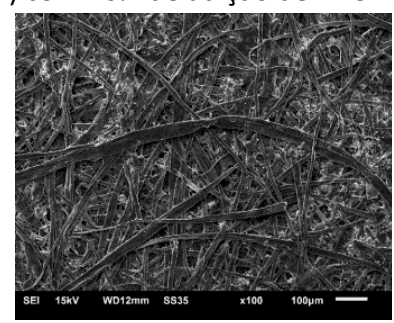

Figura 2. Microscopia eletrônica das fibras aumento 100 vezes / escala $100 \mu \mathrm{m}$, dos papéis branqueados
(A) sem adição de NFC

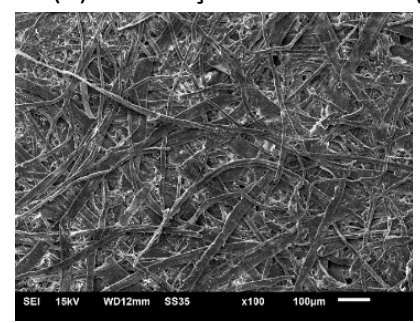

(B) com $16 \%$ de adição de NFC

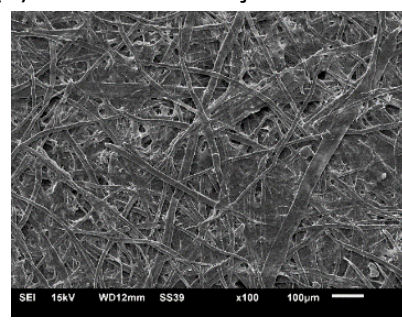

Figura 3. Microscopia eletrônica das fibras aumento 100 vezes / escala $100 \mu \mathrm{m}$, papéis não branqueados kraft.

Conforme ilustram as Figuras 2 e 3, as imagens 
microscópicas detalham que a concentração de $16 \%$ de nanofibrilas influenciou na microestrutura das folhas de papéis. É possível perceber que a partir de uma comparação entre as Figuras 2 e 3, há diferença entre os espaços vazios e preenchidos entre fibras de celulose, estes espaços foram preenchidos pelas nanofibrilas nos papéis com $16 \%$ de concentração de nanofibrilas, causando maiores quantidades de ligações entre fibras e nanofibrilas.

Viana (2013) afirma que as propriedades mecânicas do papel dependem diretamente das ligações interfibras, sendo assim, quanto menor o tamanho das fibras, maior é o potencial de ligações interfibrilares e a área de ligação entre elas

\section{Grau Schopper Riegler}

Em relação ao grau Schopper Riegler ( ${ }^{\circ} \mathrm{SR}$ ) dos papéis reciclados com fibras de papel não branqueado kraft $\mathrm{e}$ fibras de papel branqueado, demostraram valores sem e com adição de celulose nanofibrilada que estão apresentados na Tabela 3.

Tabela 3. Valores médios para grau Schopper Riegler ( $\left.{ }^{\circ} \mathrm{SR}\right)$ de fibras recicladas de papel não branqueado kraft e papel branqueado, com diferentes concentrações de NFC.

\begin{tabular}{ccc}
\hline$\%$ & Branqueado $^{\circ} \mathbf{S R}$ & Kraft $^{\circ} \mathbf{S R}$ \\
\hline 0 & $19 \mathrm{~b}$ & $6,5 \mathrm{a}$ \\
2 & $15 \mathrm{a}$ & $12,25 \mathrm{~b}$ \\
4 & $19 \mathrm{~b}$ & $16,25 \mathrm{c}$ \\
8 & $22,5 \mathrm{c}$ & $20 \mathrm{~d}$ \\
16 & $29 \mathrm{~d}$ & $23 \mathrm{e}$ \\
32 & $57,25 \mathrm{e}$ & $38,5 \mathrm{f}$ \\
64 & $82,5 \mathrm{f}$ & $88,75 \mathrm{~g}$ \\
\hline
\end{tabular}

Os valores de grau Schopper Riegler ( ${ }^{\circ} \mathrm{SR}$ ) para os tratamentos sem adição de NFC, variaram de $19^{\circ}$ para os papéis proveniente de aparas de papel branqueado e de $6,5^{\circ}$ para os papéis de aparas papel não branqueado kraft. Os valores apresentaram acréscimo devido à adição das NFC à polpa celulósica em praticamente todos os níveis de concentração de nanofibrila, apenas para o papel branqueado com $2 \%$ de NFC que obteve um decréscimo no 'SR, explicado pela reduzida quantidade de nanofibrilas adicionadas neste tratamento as quais acabam passando pela tela de desaguamento do equipamento, causando perda de massa o que gera aumento de velocidade de água escoada, que resultou no menor valor de ${ }^{\circ} \mathrm{SR}$.

Os valores ${ }^{\circ} \mathrm{SR}$ para a maior concentração de $64 \%$ para papel branqueado e não branqueado kraft, chegaram até $82,5^{\circ}$ e $88,75^{\circ}$ respectivamente. A mesma tendência foi observada por Potulski et al. (2018), que em seu estudo obteve valores de ${ }^{\circ} \mathrm{SR}$ de 72 e $62^{\circ}$ para papéis de Eucalyptus e Pinus respectivamente com adição de $9 \%$ de nanofibrilas e 2 passes no moinho.

O ${ }^{\circ} \mathrm{SR}$ está inversamente relacionado a drenabilidade da polpa celulósica, no qual o aumento no Grau Schopper indica a redução da capacidade de drenagem da polpa. Os maiores ganhos em resistência à drenagem podem estar relacionados ao fato da celulose nanofibrilada promover a maior quantidade de ligações de hidrogênio com as moléculas de água, por causa de sua superfície da parede celular mais exposta, e assim contribuir para uma maior hidratação, consequentemente maior flexibilidade e melhor arranjo das fibras na formação da folha, o que influenciar consideravelmente as propriedades físicomecânicas do papel (POTULSKI et al., 2018).

\section{Propriedades físicas e mecânicas dos papéis provenientes de fibras recicladas de papel branqueado}

Na Tabela 4 estão apresentados os valores médios encontrados para as propriedades físicas e mecânicas avaliadas dos papéis de fibras recicladas de papel branqueado com adição de celulose nanofibrilada.

Tabela 4. Valores médios das propriedades físicas e mecânicas dos papéis provenientes de fibras recicladas de papel branqueado.

\begin{tabular}{ccccccc}
\hline$\%$ & $\mathbf{G}$ & $\mathbf{T}$ & $\mathbf{E L}$ & $\mathbf{E T}$ & $\mathbf{G Y}$ & $\mathbf{E P}$ \\
\hline 0 & $46,65 \mathrm{~b}$ & $24,2 \mathrm{a}$ & $2,5 \mathrm{a}$ & $1,4 \mathrm{a}$ & $3,9 \mathrm{a}$ & $200,6 \mathrm{~b}$ \\
\hline 2 & $44,62 \mathrm{a}$ & $29,1 \mathrm{~b}$ & $3,5 \mathrm{~b}$ & $1,7 \mathrm{ab}$ & $5,4 \mathrm{a}$ & $181,9 \mathrm{a}$ \\
4 & $53,32 \mathrm{c}$ & $31,4 \mathrm{~b}$ & $3,7 \mathrm{~b}$ & $2,0 \mathrm{bc}$ & $10,3 \mathrm{ab}$ & $209,8 \mathrm{~cd}$ \\
\hline 8 & $53,95 \mathrm{~d}$ & $35,2 \mathrm{c}$ & $4,2 \mathrm{c}$ & $2,6 \mathrm{~cd}$ & $18,3 \mathrm{ab}$ & $201,7 \mathrm{~b}$ \\
16 & $54,53 \mathrm{e}$ & $40,9 \mathrm{~d}$ & $4,7 \mathrm{~d}$ & $2,9 \mathrm{de}$ & $33,3 \mathrm{~b}$ & $213,7 \mathrm{~d}$ \\
\hline 32 & $56,36 \mathrm{f}$ & $45,5 \mathrm{e}$ & $5,9 \mathrm{e}$ & $3,3 \mathrm{e}$ & $148,8 \mathrm{c}$ & $211,9 \mathrm{~d}$ \\
64 & $57,75 \mathrm{~g}$ & $64,5 \mathrm{f}$ & $7,7 \mathrm{f}$ & $5,7 \mathrm{f}$ & $1032,5 \mathrm{~d}$ & $204,8 \mathrm{bc}$ \\
\hline
\end{tabular}

$\mathrm{GR}=$ gramatura $\left(\mathrm{g} / \mathrm{cm}^{2}\right) ; \mathrm{T}=$ tração $(\mathrm{kNm} / \mathrm{kg}) ; \mathrm{EL}=$ elongamento (\%); ET = estouro $\left(\mathrm{kPa} \cdot \mathrm{m}^{2} / \mathrm{g}\right) ; \mathrm{GY}=$ Gurley $(\mathrm{Seg} / 100 \mathrm{ml}) ; \mathrm{EP}=$ espessura ( $\mathrm{mm}$ ); Médias não seguidas por uma mesma letra minúscula, na vertical, diferem estatisticamente a $5 \%$ de probabilidade pelo teste LSD Fisher.

Pode-se observar que houve uma grande diferença entre os valores de gramatura encontrados nos papéis branqueado, variando de $44,62 \mathrm{~g} / \mathrm{cm}^{2}$ até $57,75 \mathrm{~g} / \mathrm{cm}^{2}$, sendo explicado, pela forma em que a polpa foi acondicionada, não sendo um local apropriado que possibilitasse a agitação, e a coleta homogênea da mistura 
de fibras durante a retirada de amostra para a produção das folhas.

A provável justificativa para que a tendência observada para o índice de estouro tenha sido semelhante à observada para a índice de tração é que as duas propriedades sejam afetadas pelos mesmos fatores, principalmente, a capacidade de ligação entre as fibras, o principal entre eles, que foi melhorada com a adição das NFCs, gerando maior ligação entre as fibras.

Os valores encontrados pelo teste de tração cresceram, conforme o aumento da concentração de nanofibrilas, para o papel reciclado partindo de aparas de papel branqueado apresentou $24,2 \mathrm{kNm} / \mathrm{kg}$, e o papel com $64 \%$ de adição de NFC apresentou $64,5 \mathrm{kNm} / \mathrm{kg}$, sendo assim um aumento significativo nos valores de índice de tração. Potulski et al. (2018), encontrou em seu estudo valores que partiram de $28,5 \mathrm{kNm} / \mathrm{kg}$ para papéis provenientes de fibras recicladas de Eucalyptus sp., com a adição de $9 \%$ de NFC e 2 passes no moinho, encontrou 46,8 kNm/kg de indice de tração para esses papéis e sugerindo que nanocelulose é um potencial aditivo para papel.

Para o índice de estouro foi obtido um aumento conforme a adição das maiores concentrações das NFCs, partindo inicialmente com $1,38 \mathrm{kPa} \cdot \mathrm{m}^{2} / \mathrm{g}$ para o papel reciclado de fibras secundarias, até $5,7 \mathrm{KPa} . \mathrm{m}^{2} / \mathrm{g}$ para o papel reciclado com $64 \%$ de concentração de NFC. Em seu estudo, Potulski et al. (2018) encontrou para o índice de arrebentamento ou estouro para os papéis com adição de nanocelulose valores médios variando de 2,3 a 4,1 $\mathrm{kPam}^{2}$.g-1, para os tratamentos Eucalyptus sp. reciclado sem incorporação e Eucalyptus sp. reciclado com adição de 9\% de celulose nanofibrilada obtida com 2 passes pelo moinho. Schulz (2019), em seu estudo com produção de papéis com fibras recicladas de Eucalyptus, constatou valores de resistências a tração de 23,47 (KN. m/kg), Elongamento 2,42 (\%), resistência ao estouro 1,564 $\left(\mathrm{KPa} \cdot \mathrm{m}^{2} / \mathrm{g}\right)$ e relatou que as propriedades têm relações diretamente proporcionais entre elas.

Os valores encontrados para o teste de passagem do ar Gurley, obtiveram aumento significativo do papel reciclado de fibras secundarias, partindo de $3,9 \mathrm{seg} / 100 \mathrm{ml}$, chegando até $1038,5 \mathrm{seg} / 100 \mathrm{ml}$ com o papel com concentração de $64 \%$ de NFC.

Os valores médios encontrados para a espessura do papel não seguiram o mesmo padrão de acréscimo das outras propriedades conforme o aumento da concentração das NFC, está variação está diretamente ligada com gramatura encontrada para os papéis, que também não foram uniformes, está variação acarretou em valores distintos encontrados para a espessura.

\section{Propriedades físicas e mecânicas dos papéis provenientes de fibras recicladas de papel não branqueado kraft}

Na Tabela 5, estão apresentados os valores médios encontrados para as propriedades físicas e mecânicas avaliadas, dos papéis de fibras recicladas de papel não branqueado kraft com adição de celulose nanofibrilada.

Tabela 5. Valores médios das propriedades físicas e mecânicas dos papéis provenientes de fibras recicladas de papel não branqueado kraft.

\begin{tabular}{ccccccc}
\hline$\%$ & $\mathbf{G}$ & $\mathbf{T}$ & $\mathbf{E L}$ & $\mathbf{E T}$ & $\mathbf{G Y}$ & $\mathbf{E P}$ \\
\hline 0 & $55,91 \mathrm{~d}$ & $15,2 \mathrm{a}$ & $1,6 \mathrm{a}$ & $1,1 \mathrm{a}$ & $4,8 \mathrm{a}$ & $240,1 \mathrm{c}$ \\
2 & $44,17 \mathrm{a}$ & $20,9 \mathrm{~b}$ & $2,3 \mathrm{~b}$ & $1,3 \mathrm{ab}$ & $6,2 \mathrm{a}$ & $189,6 \mathrm{a}$ \\
\hline 4 & $63,27 \mathrm{~g}$ & $21,6 \mathrm{~b}$ & $2,8 \mathrm{~b}$ & $1,4 \mathrm{bc}$ & $14,6 \mathrm{a}$ & $248,3 \mathrm{c}$ \\
\hline 8 & $49,16 \mathrm{c}$ & $27,1 \mathrm{c}$ & $3,5 \mathrm{c}$ & $1,6 \mathrm{c}$ & $26,7 \mathrm{a}$ & $198,2 \mathrm{a}$ \\
\hline 16 & $48,29 \mathrm{~b}$ & $33,5 \mathrm{~d}$ & $3,9 \mathrm{~cd}$ & $2,2 \mathrm{~d}$ & $84,1 \mathrm{a}$ & $189,8 \mathrm{a}$ \\
\hline 32 & $61,22 \mathrm{f}$ & $39,5 \mathrm{e}$ & $4,4 \mathrm{~d}$ & $2,9 \mathrm{e}$ & $255,7 \mathrm{~b}$ & $225,8 \mathrm{~b}$ \\
64 & $60,91 \mathrm{e}$ & $57,0 \mathrm{f}$ & $5,5 \mathrm{e}$ & $4,4 \mathrm{f}$ & $938,8 \mathrm{c}$ & $217,6 \mathrm{~b}$ \\
\hline
\end{tabular}

$\mathrm{GR}=$ gramatura $\left(\mathrm{g} / \mathrm{cm}^{2}\right) ; \mathrm{T}=$ tração $(\mathrm{kNm} / \mathrm{kg}) ; \mathrm{EL}=$ elongamento (\%); ET = estouro $\left(\mathrm{kPa} \cdot \mathrm{m}^{2} / \mathrm{g}\right) ; \mathrm{GY}=$ Gurley $(\mathrm{Seg} / 100 \mathrm{ml}) ; \mathrm{EP}=$ espessura ( $\mathrm{mm}$ ); Médias não seguidas por uma mesma letra minúscula, na vertical, diferem estatisticamente a $5 \%$ de probabilidade pelo teste LSD Fisher.

Foi observada uma diferença significativa entre os valores de gramatura encontrados para os papéis não branqueado kraft, variando de $44,17 \mathrm{~g} / \mathrm{cm}^{2}$ até 61,22 $\mathrm{g} / \mathrm{cm}^{2}$, podendo ser explicado, pela dificuldade de agitação homogênea da mistura de fibras durante a retirada de amostra para a produção das folhas.

Ao comparar os valores apresentados na Tabela 6, as propriedades de índice de tração, elongamento, resistência ao estouro, resistência à passagem do ar (Gurley), observa-se que os papéis formados a partir de aparas de papel não branqueado kraft, obtiveram aumento significativo conforme o aumento da concentração das nanofibrilas, tendência esperada pelo crescimento do oSR, conforme o aumento da concentração das NFCs, seguindo assim o reflexo deste nas propriedades mecânicas estudadas.

Os resultados obtidos pelo teste de tração apresentaram tendência de crescimento, em função do aumento na concentração de nanofibrilas, o papel reciclado partindo de fibras secundarias de papéis não branqueados kraft apresentou $15,2 \mathrm{kNm} / \mathrm{kg}$, e o papel com $64 \%$ de adição de NFC apresentou $57,0 \mathrm{kNm} / \mathrm{kg}$, sendo assim um aumento significativo nos valores de índice de tração. Potulski et al. (2018) encontrou em seu estudo valores que partiram de $27,9 \mathrm{kNm} / \mathrm{kg}$ para papéis 
provenientes de fibras recicladas de Pinus spp., e com a adição de $9 \%$ de NFC e 2 passes no moinho, encontrou 41,1 $\mathrm{kNm} / \mathrm{kg}$ de índice de tração para esses papéis.

$\mathrm{O}$ teste de elongamento resultou em valores com tendência de crescimento conforme o aumento da concentração de nanofibrilas, partindo de 1,6\%, para o papel reciclado de aparas de papel não branqueado, chegando até 5,5\% de elongamento, aumento de 3,92\% para o papel reciclado com $64 \%$ de concentração de NFC.

$O$ índice de estouro também obteve igualmente acréscimo conforme o aumento das NFC, partindo inicialmente com $1,1 \mathrm{kPa} . \mathrm{m}^{2} / \mathrm{g}$ para o papel reciclado de fibras secundarias, até $4,4 \mathrm{KPa} . \mathrm{m}^{2} / \mathrm{g}$ para o papel reciclado com $64 \%$ de concentração de NFC.

Para o teste de passagem do ar de Gurley, foi encontrado um aumento significativo dos valores para o papel reciclado de fibras secundarias, partindo de 4,8 $\mathrm{seg} / 100 \mathrm{ml}$, chegando até $938 \mathrm{seg} / 100 \mathrm{ml}$ com o papel com concentração de $64 \%$ de NFC. Esta propriedade de resistência a passagem do ar o que corresponde a quantidade de materiais de pequenas dimensões, que ocupam os pequenos espaços entre as fibras, a passagem do ar é dificultada.

Os papéis com maiores gramaturas apresentaram maiores valores de espessura, as gramaturas dos papéis com $32 \%$ e $64 \%$ (61 e $60 \mathrm{~g} / \mathrm{cm}^{2}$ respectivamente) foram as mais uniformes com 225,8 e $217,6 \mathrm{~mm}$ respectivamente, apresentando valores semelhantes de espessura, os papéis com $2 \%$ de NFC apresentaram os piores valores de gramatura com $44,17 \mathrm{~g} / \mathrm{cm}^{2}$ e apresentou os menores valores de espessura $189,6 \mathrm{~mm}$, os papéis com $4 \%$ de NFC apresentaram espessura de $248,3 \mathrm{~mm}$, o maior valor encontrado, explicado pelo fato de sua gramatura ser a maior, com $63 \mathrm{~g} / \mathrm{cm}^{2}$.

\section{CONCLUSÕES}

Os resultados obtidos no presente estudo demonstram a influência da adição das nanofibrilas de celulose nas propriedades físicas e mecânicas nos dois tipos de papéis estudados (fibra virgem e reciclada).

A micrografia obtida pela caracterização por microscopia eletrônica de varredura dos papéis com $16 \%$ de NFC, mostrou o preenchimento das nanofibrilas nos espaços vazios dos papéis.

Os valores de grau Schopper Riegler ( ${ }^{\circ} \mathrm{SR}$ ) obtiveram acréscimo conforme adição de maiores concentrações de NFCs para ambos papéis estudados, esse aumento refletiu nas propriedades avaliadas.

Os valores de índice de tração e de estouro apresentaram um aumento significativo com maiores concentrações das NFCs adicionadas para os dois tipos de fibras avaliadas, esse acréscimo pode ser explicado pela maior ligação entre as fibras proporcionado pelas nanofibrilas de celulose, seguindo a mesma tendência para os testes de elongamento e resistência ao ar.

Os valores encontrados para a espessura do papel não seguiram o mesmo padrão de acréscimo das outras propriedades conforme o aumento da concentração das NFC, sendo influenciada pela gramatura, que foi afetada pela dificuldade de homogeneização da polpa na retirada.

A concentração que se mostrou mais positiva para ambos papéis foi com $16 \%$ de adição de nanofibrila de celulose, por apresentar ganhos nas propriedades físicas e mecânicas sem elevar os valores de permeância ao ar ao ponto de causar dificuldades de drenagem no processo industrial.

\section{REFERÊNCIAS}

AARSTAD, O. et al. Mechanical properties of composite hydrogels of alginate and cellulose nanofibrils. Polymers, v.9, n.8, p.1-19, 2017.

BIERMANN, C.J. Handbook of pulping and papermaking. 2.ed. California: Academic Press, 1996.

BRACELPA - ASSOCIAÇÃO BRASILEIRA DE CELULOSE E PAPEL. Considerações gerais sobre a atividade de reciclagem de papel no Brasil. 3o Seminário de Avaliação de Experiências Brasileiras de Coleta Seletiva de Lixo, p.14, 2014.

CASTANHO, C.G.; OLIVEIRA, R.C. Estudos de aproveitamento de rejeito fibroso industrial da polpação kraft de Eucalipto na produção de papéis reciclados. Congresso Internacional de Celulose e Papel, 2000.

HAI, L.V. et al. Effect of PFI mill and Valley beater refining on cellulose degree of polymerization, alpha cellulose contents, and crystallinity of wood and cotton fibers. Journal of Korea TAPPI, v.45, n.4, p.27-33, 2013.

ISO 1924-2 e 3: Paper and board - Determination of tensile properties. 2008.

ISO 2758: Paper - Determination of bursting strength. 2014.

ISO 5263-1: Pulps -- Laboratory wet disintegration -- Part 1: Disintegration of chemical pulps. 2004.

ISO 5263-2: Pulps - Laboratory wet disintegration. 2004.

ISO 187: Paper, board and pulps - Standard atmosphere for conditioning and testing and procedure for monitoring the atmosphere and conditioning of samples.1990.

ISO 534: Paper and board - Determination of thickness, density and specific volume. 2005. 
ISO 536: Paper and board - Determination of grammage. 1995.

ISO 5636-5: Paper and board - Determination of air permeance. 2013.

ISO 5267-1: Pulps - Determination of drainability - Part 1: Schopper-Riegler method. 1999.

ISO 5269-1: Pulps - Preparation of laboratory sheets for physical testing. 2006.

MANFREDI, M. et al. Melhoramento das propriedades de papéis reciclados através da ultrassonificação das fibras e adição de xilanas. Revista Árvore, v.36, n.4, p.777-785, 2012.

POTULSKI, D.C. et al. Influência da incorporação de celulose microfibrilada nas propriedades de resistência mecânicas do papel. Scientia Forestalis, v.42, n.103, p.345-351, 2014.

POTULSKI, D.C. et al. Nanocelulose aplicada como reforço para papel reciclado. Scientia Forestalis, v.46, n.119, p.495-505, 2018.

SCHULZ, H.R. et al. Caracterização de propriedades tecnológicas de papéis de fibra virgem e reciclada após refinamento. BIOFIX Scientific Journal, v.4, n.2, p.160-165, 2019.

SEHAQUI, H. et al. Wood cellulose biocomposites with fibrous structures at micro- and nanoscale. Composites Science and Technology, v.71, n.3, p.382-387, 2011.

VIANA, L.C. Desenvolvimento de filmes celulósicos nanoestruturados a partir da polpa kraft de Pinus sp. 2013. 125p. (Tese Doutorado).

Recebido em 13-07-2019 Aceito em 18-09-2019 